\title{
Tests of coordinate transfer from magnetic to mechanical reference for LCLS undulator fiducialization
}

\author{
Yu.Levashov and Z. Wolf \\ Stanford Linear Accelerator Center, \\ Stanford University, Stanford, CA 94309
}

April 22, 2005

\begin{abstract}
Fiducialization of the LCLS undulators will be based on magnetic measurements by Hall probe. Pointed magnets, proposed by I.Vasserman[1] for quadrupole lens fiducialization will be used as an intermediate reference. A prototype of the pointed magnet fixture has been made and tested. In this note we will describe a procedure for measuring the position of the center of the Hall probe sensitive area with respect to the undulator fiducial marks. The pointed magnet calibration procedure, a two-point algorithm for locating the magnetic center of the fixture, and test results are presented.
\end{abstract}

\section{Introduction}

The fiducialization goal is to find a location of the undulator magnetic axis (beam trajectory at a desirable $\mathrm{K}$ value) with respect to external mechanical reference marks, in our case tooling balls. The tooling balls will be used as a reference for undulator alignment in the tunnel. Very high tolerances for the undulator alignment in $Y$ direction[2],[4] limit fiducialization errors to a few micrometers to leave a reasonable error budget for the alignment itself.

A 2-D Hall probe will be used for undulator magnetic measurements. Upon completion of the measurements and tuning of an undulator we will have information about the magnetic axis location but with respect to a virtual point inside the Hall probe sensitive area. Additional measurements are necessary to transfer the coordinates from magnetic to mechanical references. To make the transfer a scan with the probe in $X, Y$, and $Z$ directions to be made to position the probe into the magnetic center of the device. Surely, the Hall probe must be the same one used for undulator magnetic measurements. Accuracy of the probe positioning will be the main component of the coordinate transfer error.

Pointed magnets or "Magnetic needles" used by I. Vasserman at Argonne National Laboratory for location of the center of the Hall probe sensitive area during a quadrupole fiducialization, proven to be suitable for the high accuracy coordinate transfer. We have designed and fabricated a prototype of the pointed magnet fixture(figure 1) with two pointed magnets fixed on C-frame facing each other.

Measurements in $X$ and $Y$ directions will be made separately, therefore, two separate pairs of $12.7 \mathrm{~mm}$ dia. tooling balls are used, one pair for each direction. Mechanical center of the fixture is defined by positions of the tooling balls. Location of the magnetic one depends not only on geometry but on properties of the magnets used. Offsets between the magnetic and mechanical centers will be found during the pointed magnet calibration on a special stand using an adequate algorithm to transfer the coordinates of the Hall probe to the tooling balls. In the following sections we will describe tests made to determine the accuracy of the probe positioning and the pointed magnet calibration.

This work was performed in support of the LCLS project at SLAC 


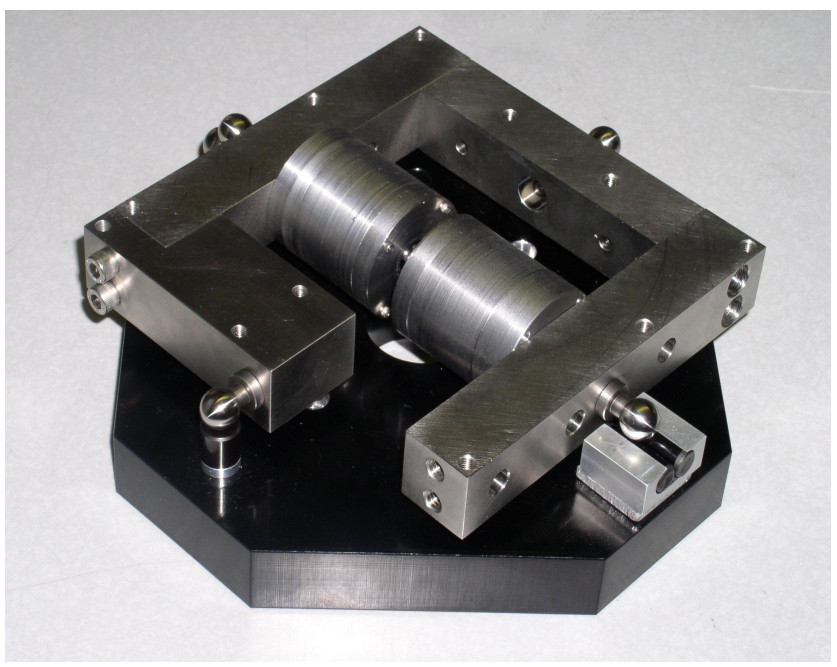

Figure 1: Pointed magnet fixture on support plate

\section{Methods and Instruments}

\subsection{Fiducialization Procedure}

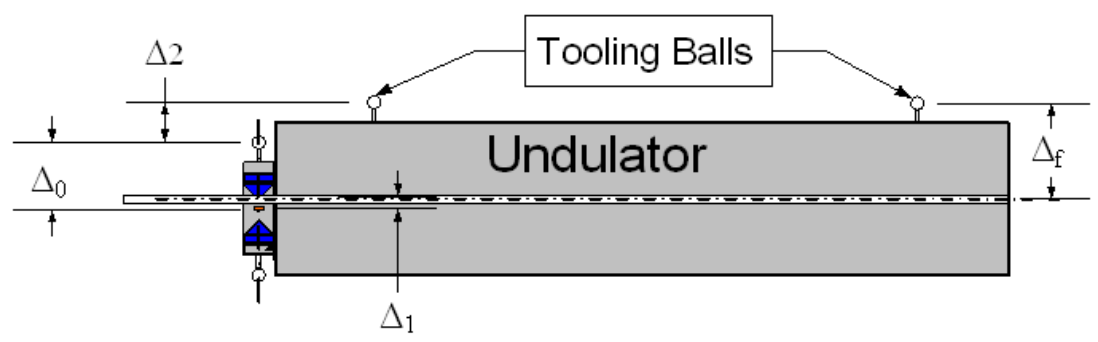

Figure 2: Fiducialization Set-Up

Undulator fiducialization will be made on the undulator measurement bench[3]. Geometry of the fiducialization set-up is shown in fig.2 (only $Y$ - direction is shown on the picture). After the completion of the undulator magnetic measurements, the fixture with pointed magnets will be secured at both ends of the undulator by screws. Accuracy of $200 \mu \mathrm{m}$ in attaching the magnets will be enough to perform the fiducialization. The Hall probe could be translated from the undulator axes to the center of the pointed magnets by motorized stages. Offsets in $X, Y$, and $Z$ directions between the undulator tooling balls and the magnetic axis of the undulator $\Delta_{f}^{x, y, z}$ will be determined in the following steps:

- Calibrate the pointed magnets fixture to find the center of the Hall probe sensitive area relative to the fixture tooling balls $\left(\Delta_{0}\right)$.

- Measure and tune the undulator. Move the Hall probe into a position which gives a desirable $K$ value.

- $\quad$ Attach the fixture to the undulator. After scanning in $X, Y$, and $Z$ directions, place the Hall probe in the center of the pointed magnets (zero field point). Offsets between the undulator axis and the center of the pointed magnets $\left(\Delta_{1}\right)$ could be read from stage encoders. 
- $\quad$ Take the whole assembly to the CMM and measure the position of the pointed magnet tooling balls with respect to the undulator fiducials $\left(\Delta_{2}\right)$.

- Apply offsets to calculate the position of the ideal beam axis with respect to the undulator fiducials. The offset $\Delta_{f}$ will be a sum of individual components: $\Delta_{f}=\sum_{i} \Delta_{i}$.

We expect to have offset $\Delta_{2}$ be measured on CMM with accuracy of $\sim 1 \mu m$. The positioning error of the stages is of same order $\sim 1 \mu \mathrm{m}$. Accuracy of the offsets $\Delta_{0}$ and $\Delta_{1}$ depends on Hall probe zero positioning and pointed magnets calibration errors, which will be discussed below.

\subsection{Pointed magnets calibration}

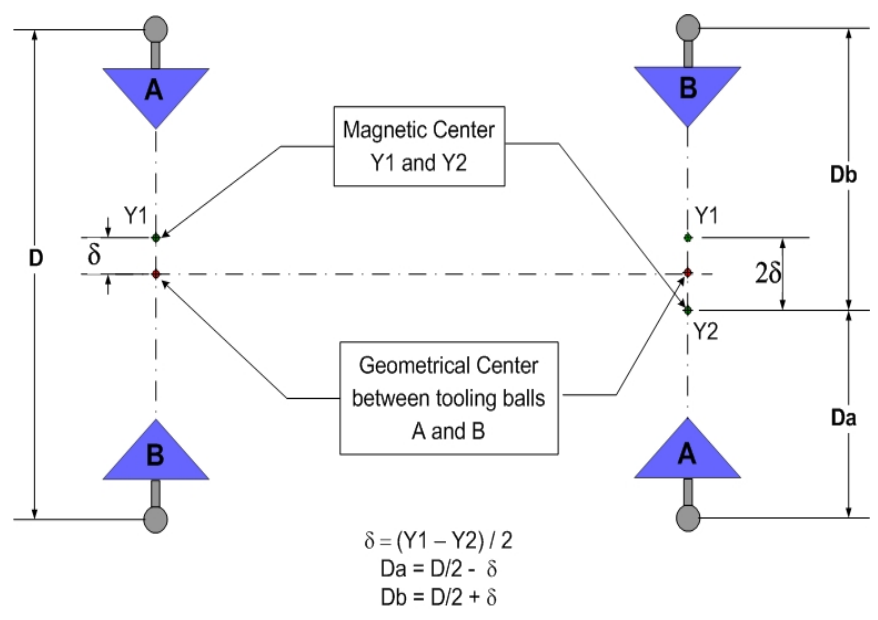

Figure 3: Calibration Algorithm

The pointed magnets could be arranged in two different ways, at $90^{\circ}$ or to face each other $\left(180^{\circ}\right)$. We have tried both cases but in the first one magnetic field from a pointed magnet extended too far and interfered with magnetic field from the second one. There was no zero point available for measurements. In addition, misalignment of the magnets will be a main source of errors. Having no reliable algorithm to implement the $90^{\circ}$ scheme we have chosen $180^{\circ}$ magnet arrangement.

The idea of the calibration is shown in figure 3. Again, we consider the $Y$ direction only. The calibration consists of two steps. Magnet arrangement for the first step is shown on left part of the picture. The pointed magnet fixture is placed on a motorized stage so that one axis of the stage be parallel to the line connecting the tooling ball centers. Scanning along the line a zero field point $Y 1$ or the magnetic center of the pointed magnets will be found and the probe placed into the center. The position of the probe could be read from the stage encoder as $y_{1}$. In an ideal case, when the pointed magnets are perfectly machined and aligned, the magnetic center will be located on a line connecting the tooling ball centers but shifted at distance $\delta$ from the geometrical one due to difference in magnet's strength. The goal of the pointed magnet calibration is to measure the shift.

For the second step exchange locations of the magnets $A$ and $B ; A$ goes exactly into position of $B$ and $B$ into $A$; shown on the right side of the picture. If the distance between tooling balls is the same (no mechanical deformations), the geometrical (mechanical) center of the fixture will stay at the same place but magnetic one will shift into position $Y 2$ due to initial offset $\delta$. Move the Hall probe by the stage into magnetic center or the point $Y 2$ again. The distance the probe traveled or the shift of the magnetic center $2 \delta$ could be measured by the stage encoder. Distance $D$ between tooling balls could be measured on CMM. Location of the magnetic center $D_{a}$ or $D_{b}$ w.r.t. the tooling balls is 
easily calculated.

There are two possible ways to exchange the magnet positions: rotate them at 180 degrees or flip them over. Tests of the fixture prototype show that the results of the calibration depend on how the magnets were exchanged. A few factors could contribute to this effect: stray magnetic fields, mechanical rigidness of the fixture which results in changing of geometrical dimensions, misalignment, and non-symmetrical design of the magnets. The same factors could change the calibration which needs to be retained during the fiducialization process. Most of these factors were taken into account in the pointed magnet design.

\subsection{Pointed magnet design}

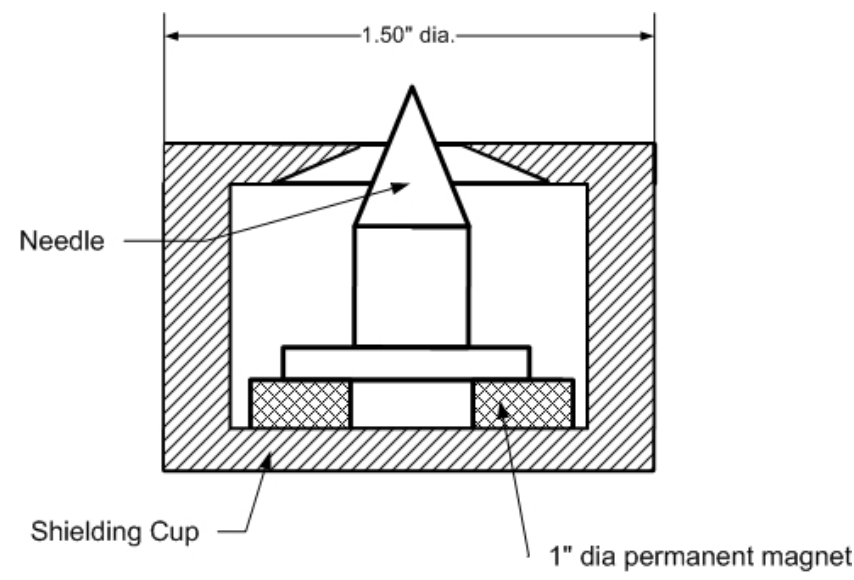

Figure 4: Pointed magnet lay-out

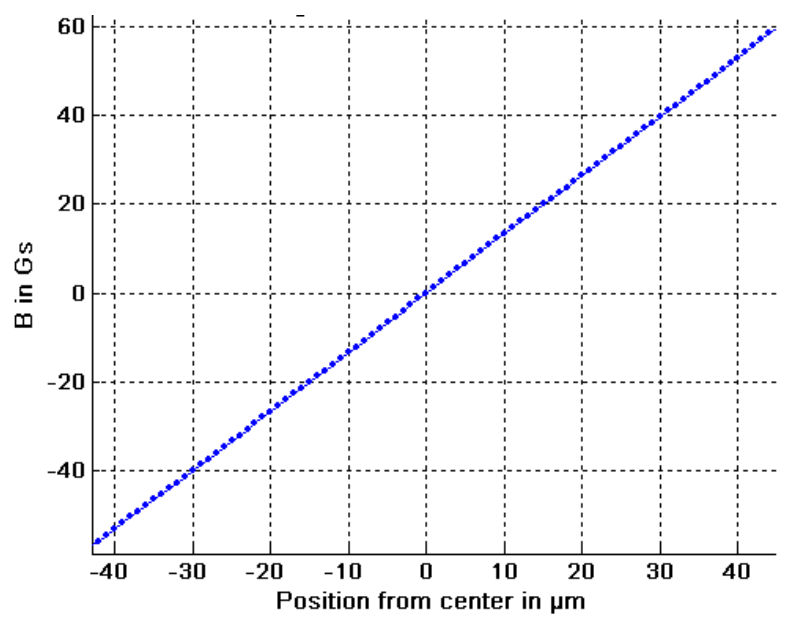

Figure 5: Magnetic field vs. distance from pointed magnet center in Y - direction

The pointed magnet assembly is simple, not expensive and consists of 1008 steel needle and $25.4 \mathrm{~mm}$ dia., $6.35 \mathrm{~mm}$ high neodymium magnet available off-shelf at on-line store. The pointed magnets were 
made axial-symmetrical with iron shielding caps around them, figure 4, which protect the area nearby the fixture magnetic center from stray fields. The thickness of the cup wall is $5 \mathrm{~mm}$; the needle tip is out off the cup by $2 \mathrm{~mm}$. Magnetic field nearby the center is almost linear, figure 5 , with a zero point well defined. The gradient of the field is $\sim 1 G / \mu m$.

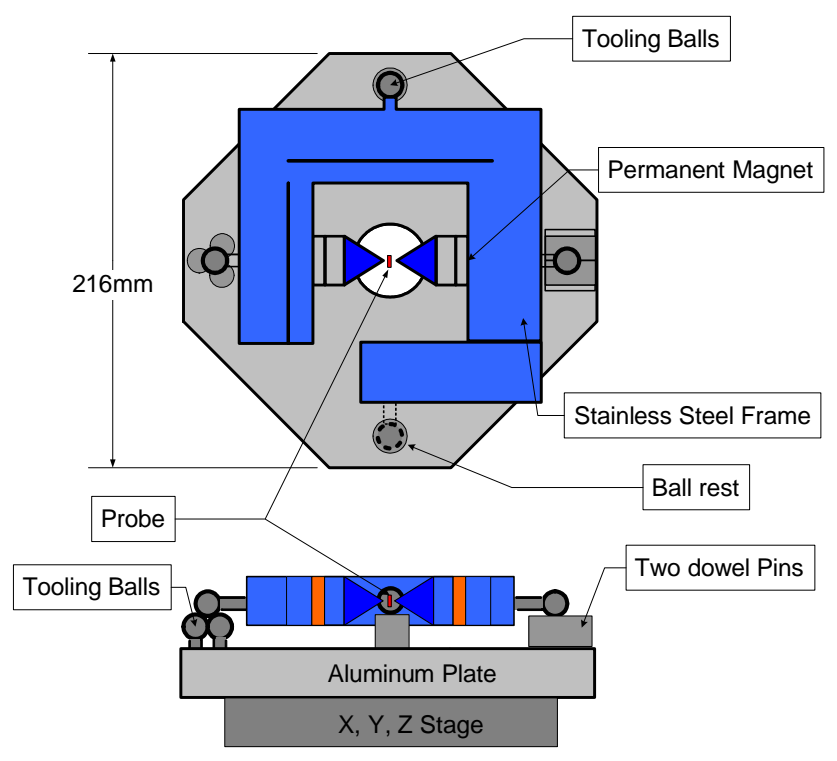

Figure 6: Pointed magnet calibration set-up

The pointed magnets fixture consists of two magnet assemblies secured on a stainless steel C-frame with 3 tooling balls pressed into it. The fourth tooling ball is mounted on a separate detachable block. It is needed for the calibration only and will be removed before securing the fixture on an undulator. Distance between the magnet tips is $7 \mathrm{~mm}$.

Pointed magnet calibration set-up is shown on figure 6. The Sentron Hall probe is fixed on a support table and comes up into the magnet. Magnets are placed on a special base plate and could be moved by motorized $x, y$ stages with $0.1 \mu \mathrm{m}$ resolution. The plate has a kinematic support for housing tooling balls to accurate and repeatable position the magnets with respect to the probe. The kinematic support was made of 3 balls instead of a cup, two dowel pins instead of $V$ - block, and two flats serve as supports. Repositioning a spherical surface on a spherical or on a cylindrical surface is reliable. Tests showed that it could be done repeatedly with $1 \mu m$ accuracy.

\subsection{Two - point measurement algorithm}

Misalignment of the pointed magnets is always the case in real life resulting in a shift of magnetic center location and, therefore, in a significant Hall probe positioning error. To reduce this error to a negligible value an adequate two-point measurement algorithm has been developed.

We will consider $2-D$ approach $(Z=$ const), since for $3-D$ case an additional scanning in $Z$-direction should be added. Suppose we have two identical magnets faced each other but misaligned, figure 7 . There will be a zero field (center) line tilted due to misalignment of the magnets. The initial probe position could be pinned with, let say, $200 \mu \mathrm{m}$ variation (somewhere inside a square $200 \times 200 \mu \mathrm{m}$ ). First, move the magnets in $Y$ direction to have one of them close to the probe, and scan in $X$ direction. A polynomial fit has been applied to the measurement results to find $X$ position of the field maximum. From the point of the field maximum $X_{\max }$ the magnets will be moved back in $Y$ direction at the 


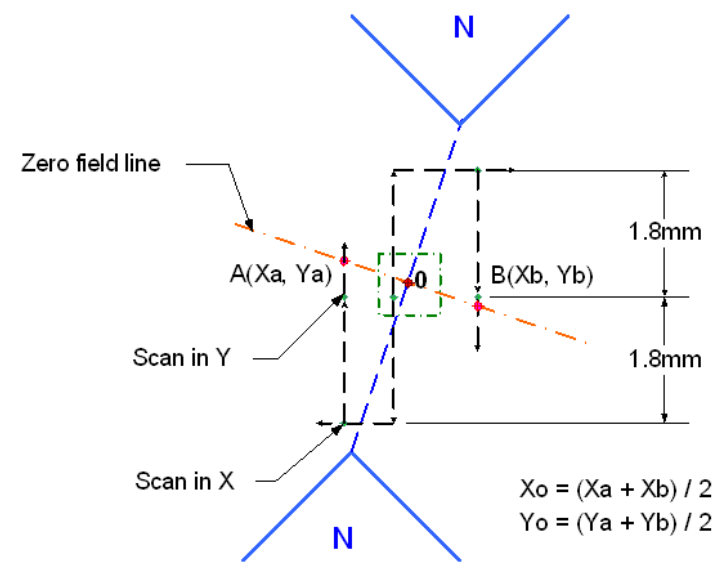

Figure 7: Two-point measurement algorithm

same distance, so that the probe will be close to the center line. By scanning from that point in $Y$ and applying a linear interpolation, a point $A$ could be found as a magnetic center of the fixture. Due to misalignment point $A$ will be offset from the real magnetic center in both $X$ and $Y$ directions, therefore, using only one magnet for the zero probe calibration will result in fiducialization error. Next, the magnets will be returned into the initial position and the same procedure repeated but for the second magnet. It gives us the position of the point $B$. We have assumed that the location of the real magnetic center is in the middle of the line connecting two points $A$ and $B$. It is not absolutely correct but results have a negligible error. In addition, the relative coordinates of points $A$ and $B$ give usable information about the magnet misalignment.

\section{Test results}

\subsection{Accuracy of Hall Probe zero positioning}

We tried to do the measurements as close as possible to the real ones on the undulator. The measurements shown were made using Sentron XZM12 Hall probe. Field readings were taken from the probe analog output by HP3457A voltmeter set for $3 \mathrm{~V}$ measurement range.

Starting from random initial positions in $\pm 200 \mu m$ range and following the two-point measurement algorithm, the probe was placed repeatedly into the center of the pointed magnets. The probe was fixed on a support table but the magnets moved by motorized stages. The results of probe zero positioning in $x$ and $y$ directions are shown on figure 8. Accuracy (standard deviation) in $x$ direction is $2 \mu \mathrm{m}$, dot line; in $y$ direction $-1 \mu \mathrm{m}$, dashed line.

\subsection{Stray magnetic fields}

The procedure of the coordinate transfer is based on zero-field measurements. Any, even very weak, stray magnetic fields will affect the results of the coordinate transfer by shifting the location of the magnetic center of the fixture. Any changes in the outside conditions (direction of earth field or presence of an iron material nearby the pointed magnets) could change the results by a non - negligible amount, causing significant fiducialization error. The problem exists for the undulator fiducialization since each undulator has shielding steel plates at both ends of it and the pointed magnet fixture will 


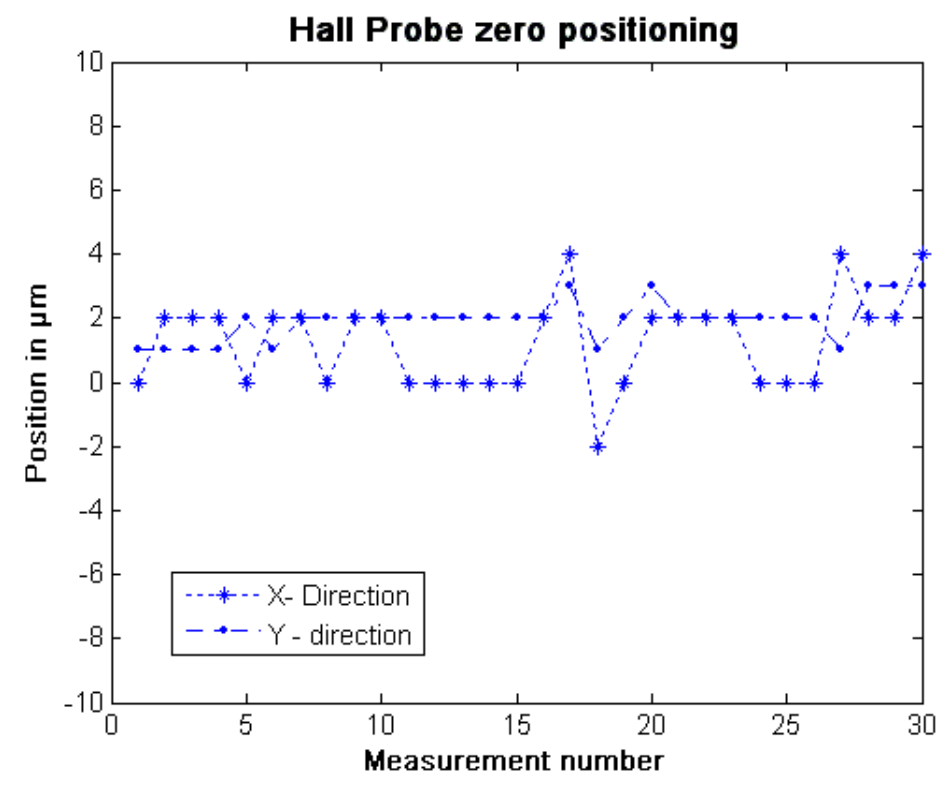

Figure 8: Results of Hall probe zero positioning: pointed magnets with shielding cups.

be mounted close to these plates. To check, how close the pointed magnets could be placed to the shielding plate, we have $18 \mathrm{~cm} \times 18 \mathrm{~cm}$ steel plate positioned at different distances from the magnets. The plate was slightly naturally magnetized $\sim 2 G$. The results of the Hall probe positioning are shown in figure 9 .

The measurements were made both without shielding cups - star markers - and with them circles. To make sure there was no temperature drift a few points were taken without the plate, at the beginning and at the end of the measurement set. Without the shielding cups the pointed magnets should be spaced at least $25 \mathrm{~cm}$ from the undulator end plate to make the positioning error insignificant. Axial-symmetrical design of the magnets and using shielding cups (figure 4) allows to place the pointed magnets as close as $3 \mathrm{~cm}$ to the plate without any noticeable effect on the center point location.

\subsection{Calibration of the pointed magnets}

Results of measurements of an offset between the magnetic and mechanical center of pointed magnets $2 \delta$ in $Y$ direction are shown in figure 10 .

The accuracy of measurements(standard deviation) is $\sim 2 \mu \mathrm{m}$. There are two sets of measurements shown. The first set was taken when the magnets were rotated 180 degrees and the second one when magnets were flipped. A shift $\Delta \approx 10 \mu \mathrm{m}$ in results of two sets of measurements could come from different sources of errors like an insufficient rigidness of the calibration fixture prototype, tooling ball surface machining errors ( $~ 5 \mu \mathrm{m}$ tolerance), difference in height between the probe and pointed magnet tips when we flip the magnets (if there is a misalignment of the magnets in $Z$-direction), etc.

\section{Discussion}

The test results show that the probe could be placed into the pointed magnets center with an accuracy of $1 \mu \mathrm{m}$ or better (in $Y$ direction as a more important one for the fiducialization) but errors in 


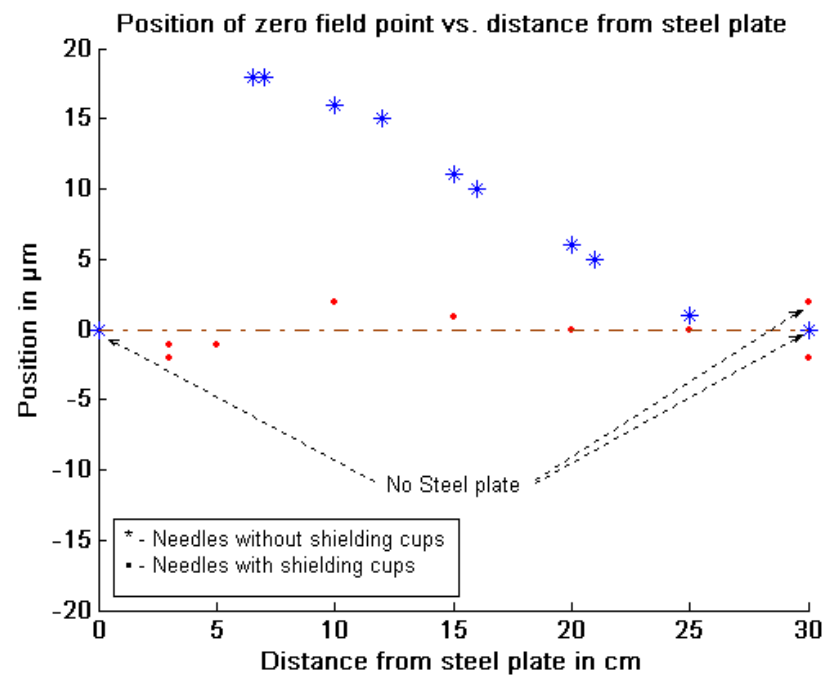

Figure 9: Position of magnetic center vs.distance from $18 \mathrm{~cm} \times 18 \mathrm{~cm}$ steel plate

positioning the magnets on the calibration fixture could decrease the accuracy of the coordinate transfer to $5 \mu \mathrm{m}$ in the worst case, which is still acceptable for the fiducialization. There are ways for improvements; make the calibration fixture more rigid, select tooling balls, have extra $z$-stage to adjust the probe position.

One could notice that during the probe calibration zero field measurements are made but the undulator magnetic measurements to be done at $1.25 \mathrm{~T}$. It is unclear if the center of probe sensitive area would not change its location inside the probe. In other words, the magnetic reference point could possibly shift in space when the probe has been moved from a zero field into a high field area. The fiducialization error due to this effect should be constant for all undulators, since they have the same magnetic field strength. As a result, undulators will be shifted from an ideal position at the same value, i.e. have a slightly different $K$. The last could be easy corrected by steering the beam at the beginning of the undulator section.

\section{Summary}

The undulator fiducialization procedure has been finalized. Calibration fixture based on pointed magnets technique has been developed, fabricated, and tested. A two-point calibration algorithm has been proposed and proven to be adequate. Accuracy of the coordinate transfer between magnetic and mechanical references is expected to be: $5 \mu \mathrm{m}$ in $Y$ direction and $10 \mu \mathrm{m}$ in $X$ which are smaller the error budget for coordinate transfer by factor of 3 .

\section{Asknowledgement}

We would be delighted to thank Isaac Vasserman and Kirsten Hacker for ideas and fruitful discussions. 


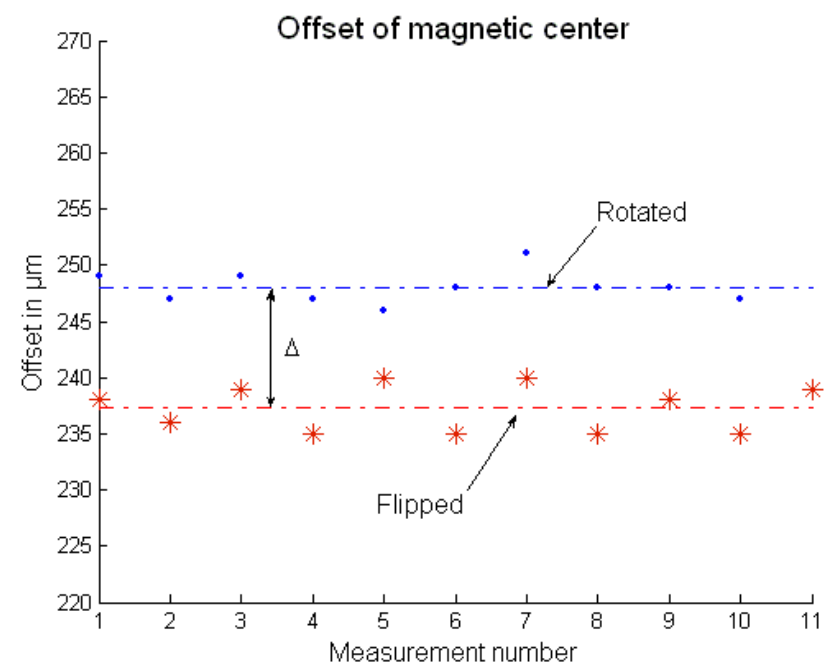

Figure 10: Results of pointed magnet calibration

\section{References}

1. Vasserman I. Quadrupole magnetic center definition using the hall probe measurement technique. APS-Pub. LS-285, Argonne, 2004

2. Heinz-Dieter Nuhn et al., "General Undulator System Requirements", LCLS Physics Requirements Document 1.4-001.

3. Wolf Z. "Requirements for the LCLS Undulator Magnetic Measurement Bench" LCLS-TN-04-8

4. R.Ruland "Installation, Alignment, Magnetic Measurement, and Fiducialization" FAC Meeting, Apr. 7-8, 2005. 